\title{
In vivo visualization of PARP inhibitor pharmacodynamics
}

\author{
Elizabeth S. McDonald, ${ }^{1}$ Austin R. Pantel, ${ }^{2}$ Payal D. Shah, ${ }^{3}$ Michael D. Farwell, ${ }^{2}$ Amy S. Clark, ${ }^{3}$
} Robert K. Doot, ${ }^{2}$ Daniel A. Pryma, ${ }^{2}$ and Sean D. Carlin ${ }^{2}$

${ }^{1}$ Division of Breast Imaging, Department of Radiology, ${ }^{2}$ Division of Nuclear Medicine Imaging and Therapy, Department of Radiology, and ${ }^{3}$ Division of Hematology/Oncology, Department of Medicine, University of Pennsylvania, Philadelphia, Pennsylvania, USA.

BACKGROUND. $\left[{ }^{18} \mathrm{~F}\right]$ FluorThanatrace $\left(\left[{ }^{18} \mathrm{~F}\right] \mathrm{FTT}\right)$ is a radiolabeled poly (adenosine diphosphateribose) polymerase inhibitor (PARPi) that enables noninvasive quantification of PARP with potential to serve as a biomarker for patient selection for PARPi therapy. Here we report for the first time to our knowledge noninvasive in vivo visualization of drug-target engagement during PARPi treatment.

METHODS. Two single-arm, prospective, nonrandomized clinical trials were conducted at the University of Pennsylvania from May 2017 to March 2020. PARP expression in breast cancer was assessed in vivo via [ $\left.{ }^{18} \mathrm{~F}\right] \mathrm{FTT}$ PET before and after initiation of PARPi treatment and in vitro via [125]] $\mathrm{KX} 1$ (an analog of [ $\left.{ }^{18} \mathrm{~F}\right] \mathrm{FTT}$ ) binding to surgically removed breast cancer.

RESULTS. Thirteen patients had baseline $\left[{ }^{18} \mathrm{~F}\right] \mathrm{FTT}$ PET. Nine of these then had resection and in vitro evaluation of $\left[{ }^{18} \mathrm{~F}\right] \mathrm{FTT}$ uptake with an analog and uptake was blocked with PARPi. Of the other 4 patients, 3 had $\left[{ }^{18} \mathrm{~F}\right] \mathrm{FTT}$ PET uptake, and all had uptake blocked with treatment with a therapeutic PARPi. Initial in vivo $\left[{ }^{18} \mathrm{~F}\right] \mathrm{FTT}$ tumor uptake ranged from undetectable to robust. Following initiation of PARPi therapy, $\left[{ }^{18} \mathrm{~F}\right] \mathrm{FTT}$ uptake was not detectable above background in all cases. In vitro tumor treatment with a PARPi resulted in $82 \%$ reduction in $\left.{ }^{[25}{ }^{12}\right] \mathrm{KX} 1$ binding.

CONCLUSION. $\left[{ }^{18} \mathrm{~F}\right] \mathrm{FTT}$ noninvasively quantifies PARP-1 expression. Early results indicate ability to visualize PARPi drug-target engagement in vivo and suggest the utility of further study to test $\left[{ }^{18} \mathrm{~F}\right]$ FTT PET as a predictive and pharmacodynamic biomarker.

RKD has received fees for consulting from Trevarx Biomedical, Inc., which holds a license for $[18 \mathrm{~F}]$ FluorThanatrace. DAP reports grants and personal fees from Siemens, grants and personal fees from 511 Pharma, personal fees from Bayer, grants and personal fees from Fusion Pharmaceuticals, grants and personal fees from Progenics Pharmaceuticals, personal fees from Actinium Pharmaceuticals, grants from Nordic Nanovector, and personal fees from Ipsen.

Copyright: (c) 2021, McDonald et al. This is an open access article published under the terms of the Creative Commons Attribution 4.0 International License.

Submitted: December 3, 2020

Accepted: March 10, 2021

Published: April 22, 2021

Reference information: /CI Insight. 2021;6(8):e146592. https://doi.org/10.1172/jici. insight.146592.

FUNDING. Metavivor Translational Research Award, Susan G. Komen for the Cure (CCR 16376362), Department of Defense BC190315, and Abramson Cancer Center Breakthrough Bike Challenge.

\section{Introduction}

Poly (adenosine diphosphate-ribose) polymerase inhibitors (PARPi) show clinical efficacy in individuals with breast cancer and BRCA1/2 mutations (1). Still, mutation status is not universally predictive of response, and some patients without germline mutations derive significant benefit $(2,3)$. Heterogeneous clinical response to PARPi, even within trials using mutation status and other homologous recombination deficiency biomarkers, creates a need for a predictive biomarker for PARPi therapy. $\left.{ }^{18} \mathrm{~F}\right] \mathrm{Fluo}-$ rThanatrace $\left(\left[{ }^{18} \mathrm{~F}\right] \mathrm{FTT}\right)$ has a chemical structure similar to therapeutic PARPi and is radiolabeled with the positron emitter, ${ }^{18} \mathrm{~F}$, for PET imaging $(4,5)$. Human dosimetry of $\left[{ }^{18} \mathrm{~F}\right] \mathrm{FTT}$ in 8 cancer patients was previously reported by Michel et al. to be comparable to other radiotracers currently in clinical use, with a table of estimated $\left[{ }^{18} \mathrm{~F}\right] \mathrm{FTT}$ residence times in organs (Supplemental Table 3 in ref. 5). $\left[{ }^{125} \mathrm{I}\right]$ $\mathrm{KX} 1$ is an analog with a longer half-life used for in vitro radioligand binding assays (6). Preclinical studies show high affinity and specificity of these tracers for PARP-1 $(6,7)$. In vitro data demonstrate that the level of PARP-1 correlates positively with cytotoxicity of PARPi and that PARP-1 expression is required for PARPi efficacy (6-9). We previously reported on imaging and quantifying PARP using $\left[{ }^{18} \mathrm{~F}\right] \mathrm{FTT}$ in breast and ovarian cancer $(7,10)$. This study builds on that body of work by demonstrating 
A

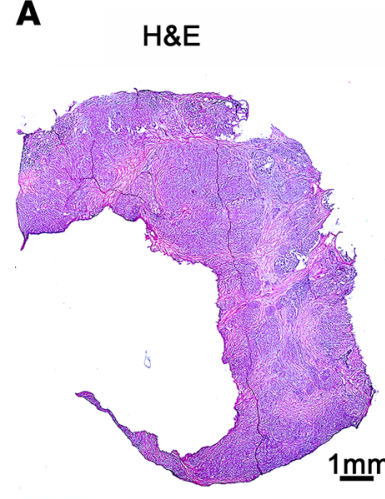

[ $\left.{ }^{125}\right] \mathrm{KX} 1$ binding

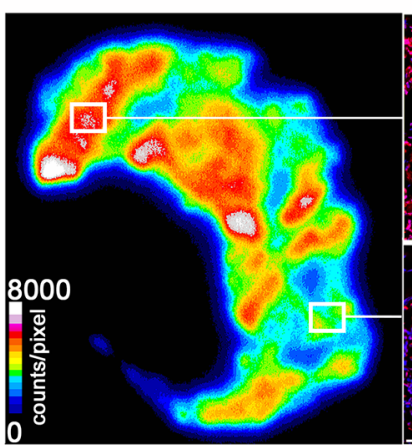

PARP1 IF (red)

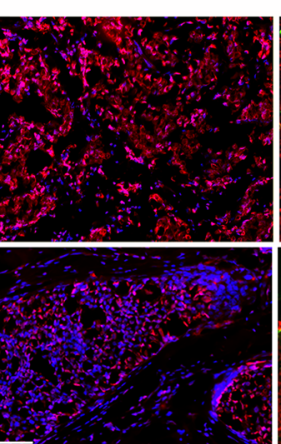

AE1/AE3 (red) CD3 (green)

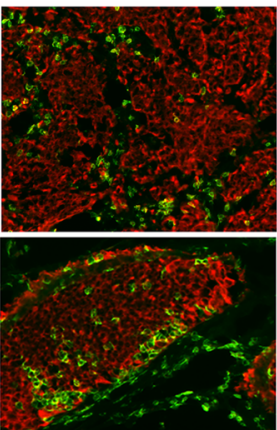

$\left[{ }^{125}\right] \mathrm{KX} 1+$ Olaparib

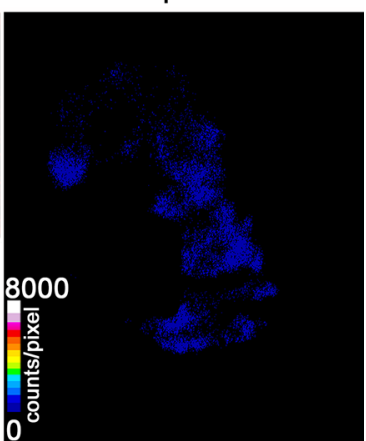

B
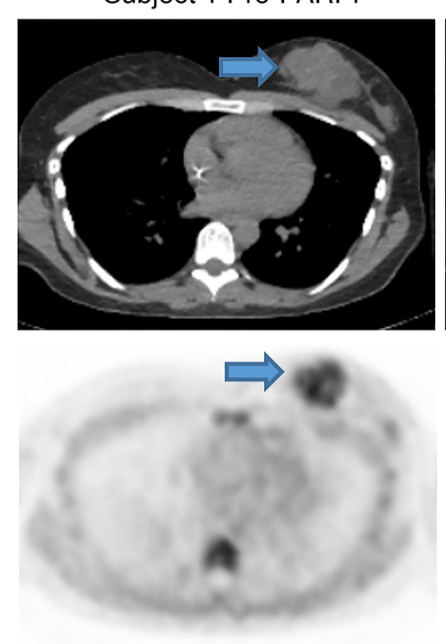

Subject 1 Post-PARPi

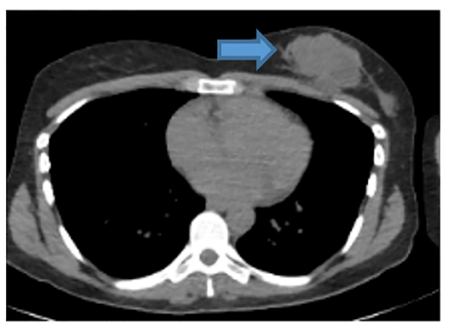

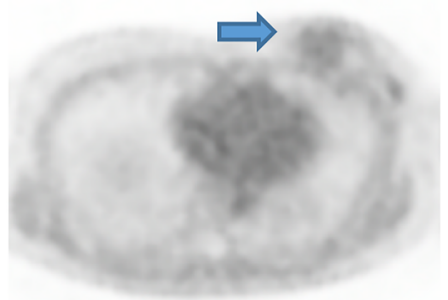

Subject 2 Pre-PARPi
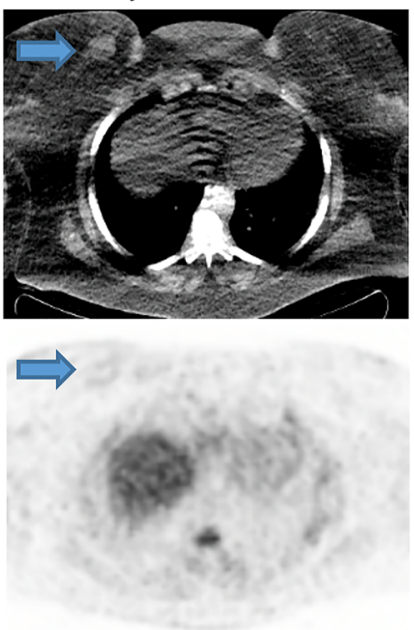

Subject 2 Post-PARPi

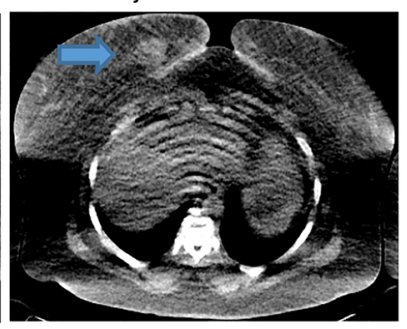

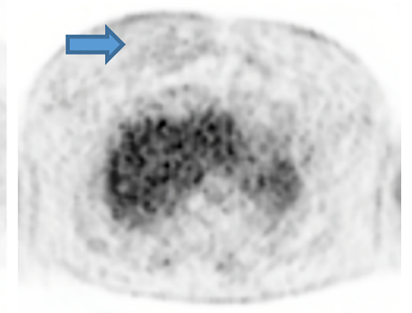

Figure 1. Baseline expression and subsequent suppression of PARP-1 after PARPi treatment in breast cancer. (A) Representative whole-tumor section demonstrates suppression of PARP radiotracer uptake with olaparib. Quantitative radioligand binding analysis (autoradiography) was followed by hematoxylin and eosin (H\&E) staining. Contiguous cryosections were used to perform chromogenic PARP-1 immunofluorescence (red) with DAPI counterstain (blue). AE1/AE3 staining was performed to discriminate epithelial tumor cells (red), and CD3 staining was used to identify tumor-infiltrating T cells (green). Autoradiography demonstrates heterogeneity of PARP-1 expression at the microscopic level with spatial concordance between the intensity of $\left[{ }^{125} \mid\right] \mathrm{KX} 1$ uptake and expression of PARP-1 measured by immunofluorescence. [ ${ }^{125}$ ]] KX1 plus $20 \mu \mathrm{M}$ olaparib on a sequential section demonstrates tracer reduction to background levels. Scale bar on whole specimen H\&E-stained slide is $1 \mathrm{~mm}$. (B) [ $\left.{ }^{18} \mathrm{~F}\right] \mathrm{FTT}$ PET/CT image taken before and approximately 1 week after PARPi treatment for 2 women with advanced triple-negative breast cancer. Subject 1 had moderate [ $\left.{ }^{18} \mathrm{~F}\right] \mathrm{FTT}$ uptake

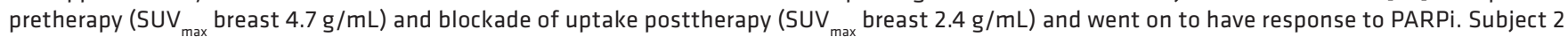
had minimal uptake pretherapy (SUV ${ }_{\max }$ breast $2.3 \mathrm{~g} / \mathrm{mL}$ ) and similar uptake posttherapy (SUV max $_{\text {mase }}$ breast $2.4 \mathrm{~g} / \mathrm{mL}$ ) and had progression on PARPi.

for the first time to our knowledge in vivo visualization of PARPi binding and subsequent PARP-1 suppression, with underlying biologic heterogeneity between tumors of similar molecular subtypes.

\section{Results}

An ex vivo PARPi competition assay was performed in tumors from 9 subjects with correlative in vivo PARP-1 imaging. There was an average of $82 \%$ (median $88 \%$, range $55 \%-99 \%$ ) suppression of $\left[{ }^{125} \mathrm{I}\right] \mathrm{KX} 1$ uptake when olaparib was added to available frozen tumor tissue as shown for a representative subject. Spatial concordance of PARP-1 intratumor heterogeneity and $\left[{ }^{125} \mathrm{I}\right] \mathrm{KX} 1$ was also demonstrated (Figure 1A).

Four study participants with stage III/IV breast cancer planning to receive PARPi treatment consented and completed pre- and post-PARPi $\left[{ }^{18} \mathrm{~F}\right] \mathrm{FTT}$ PET/CT. Age range was 41 to 71 (median 52). The histology of the primary breast malignancy was invasive ductal carcinoma for all patients. There were 3 triple-negative tumors and 1 estrogen receptor-positive tumor. Baseline PET imaging in 3 patients demonstrated moderate $\left[{ }^{18} \mathrm{~F}\right] \mathrm{FTT}$ uptake at sites of disease (maximum standardized uptake value $\left[\mathrm{SUV}_{\max }\right]$ range 4.2-6.8), with subsequent stable disease or tumor regression after PARPi. One subject did not have $\left[{ }^{18} \mathrm{~F}\right] \mathrm{FTT}$ uptake above regional background in any tumor (Figure 1B), with subsequent enlargement of the breast tumor 
and eventual distant disease and death within 1 year. After PARPi initiation, tumor $\left[{ }^{18} \mathrm{~F}\right] \mathrm{FTT}$ uptake was at background levels for all patients, with representative postimages in Figure $1 \mathrm{~B}$ and the graphical abstract. There was intrasubject heterogeneity of pretreatment uptake as demonstrated in the graphical abstract. In this study subject, pretreatment $\mathrm{SUV}_{\max }$ were 4.7 (breast), 7.7 (axillary node), 4.7 (spine), and $5.4 \mathrm{~g} / \mathrm{mL}$ (liver), and posttreatment $\mathrm{SUV}_{\max }$ were 2.4 (breast), 2.2 (axillary node), 2.3 (spine), and $2.9 \mathrm{~g} / \mathrm{mL}$ (liver).

\section{Discussion}

In breast cancer, PARPi are an important treatment option for $B R C A$-associated human epidermal growth factor receptor 2-negative metastatic disease and may soon be used in the neoadjuvant setting. Response to PARPi, though, remains variable, and a reliable predictive biomarker represents an unmet clinical need. Additionally, new, more effective drugs targeting PARP expression are in development, and an in vivo tool to quantify novel pharmacodynamics could help facilitate early evaluation of efficacy.

The data presented here leverage prior work indicating that $\left[{ }^{18} \mathrm{~F}\right] \mathrm{FTT}$ PET can measure regional PARP expression $(6,7)$ and demonstrate that uptake in breast cancer decreased to background after introducing a pharmacologic PARPi, building on in vitro assays supporting the use of $\left[{ }^{18} \mathrm{~F}\right] \mathrm{FTT}$ PET as a measure of drug-target engagement. $\left[{ }^{18} \mathrm{~F}\right] \mathrm{FTT}$ uptake at baseline signifies drug-target expression; abrogation of signal after PARPi therapy represents drug-target engagement. Both of these measures could potentially serve as biomarkers for PARPi response, and preclinical work suggests PARP-1 is required for PARPi cytotoxicity (6-9). These results provide proof-of-concept rationale that target engagement during PARPi treatment can be visualized and suggest the potential usefulness of [18F]FTT PET as a response biomarker and tool to visualize pharmacodynamic effects of PARPi compounds. Future studies will investigate the predictive value of this tracer for PARP therapy and whether the observed heterogeneity of initial uptake or uptake suppression after treatment corresponds to mixed response at specific sites of disease.

\section{Methods}

Study design. Study participants were recruited at the University of Pennsylvania for NCT03846167 July 2019 to March 2020. Inclusion criteria were biopsy-proved breast cancer, consent for tissue analysis, and willingness to undergo $\left[{ }^{18} \mathrm{~F}\right] \mathrm{FTT}$ PET/CT before and after PARPi treatment. Four potential subjects were approached for the pilot study, provided written informed consent, and participated in the trial. Nine additional participants, described previously (10), underwent a [ $\left.{ }^{18} \mathrm{~F}\right] \mathrm{FTT}$ PET/CT scan as part of NCT03083288 with untreated tumor collection for PARP radioligand analysis. Testing for BRCA1/2 was performed on all subjects, with 1 BRCA1 mutation identified (10).

Tissue preparation. Freshly excised surgical tissue was immediately embedded in optimal cutting temperature (Thermo Fisher Scientific) and flash-frozen in isopentane/liquid nitrogen. Tissues were stored at $-80^{\circ} \mathrm{C}$ and contiguous $10 \mu \mathrm{m}$ cryosections cut for analysis.

Immunofluorescence staining. The $10 \mu \mathrm{m}$ sections of frozen tissue were stained with rabbit anti-human PARP (clone 46D11, Cell Signaling Technology 9532, 1:1000), with secondary detection using rabbit Alexa Fluor 568 (Life Technologies, Thermo Fisher Scientific, A11036, 1:250). DAPI (Thermo Fisher Scientific $62248,0.5 \mu \mathrm{g} / \mathrm{mL}$ ) was a nuclear counterstain. Contiguous $10 \mu \mathrm{m}$ sections of frozen tissue were stained using mouse anti-human CD3 (clone LN10, Leica PA0122, undiluted) and mouse anti-human multi-cytokeratin (clones AE1 and AE3, Leica NCL-L-AE1/3, 1:400). CD3 visualization was done using Opal HRP Polymer (PerkinElmer ARH1001EA) and Opal 520 Reagent Pack (PerkinElmer FP1487001KT, 1:100). Detection of cytokeratin was done by incubating with goat anti-mouse Alexa Fluor 647 (Life Technologies, Thermo Fisher Scientific, A21236, 1:250). Leica Biosystems BOND-III (Leica Microsystems) was utilized, and heat-induced epitope retrieval with BOND Epitope Retrieval Solution 2 (Leica Microsystems AR9640) was done for 20 minutes. Slides were mounted in ProLong Gold (Life Technologies, Thermo Fisher Scientific, P36961). The primary monoclonal PARP-1 antibody was validated on fixed and frozen tissue as a strong nuclear signal across a variety of normal tissues known to express PARP-1 prior to use.

Radioligand binding assay. Contiguous $10 \mu \mathrm{m}$ cryosections identical to those used for immunofluorescence were incubated with [ $\left.{ }^{125} \mathrm{I}\right] \mathrm{KX} 1(50 \mathrm{nM})$, in the absence or presence of $20 \mu \mathrm{M}$ olaparib (Selleckchem). After 60 minutes, sections were washed, dried, and assessed for bound radioligand by exposing to a storage phosphor plate (BAS-IP SR, Thermo Fisher Scientific). Quantitative autoradiographic images were acquired using GE Healthcare Typhoon FLA 7000 plate reader and analyzed using GE Healthcare ImageQuant 8.1 software. Sequential sections were stained with $\mathrm{H} \& \mathrm{E}$ and scanned as above. 
$\left[{ }^{18}\right.$ FJFTT PET/CT imaging and analysis. $\left[{ }^{18} \mathrm{~F}\right] \mathrm{FTT}$ was synthesized at the University of Pennsylvania Cyclotron Facility as previously described (10). Study subjects were scanned on an Ingenuity TF PET/CT (Philips Healthcare). Quantitative analysis of PET images was done by a fellowship-trained nuclear medicine physician and fellowship-trained breast radiologist from a 20-minute scan beginning 60 minutes after injection using MIM v6.7 (MIM Software Inc.). There were no study-related adverse events in study participants. Tumor uptake of $\left[{ }^{18} \mathrm{~F}\right] \mathrm{FTT}$ was reported as $\mathrm{SUV}_{\max }$ in units of $\mathrm{g} / \mathrm{mL}$ as previously reported for this tracer $(7$, 10). $\mathrm{SUV}_{\max }$ was recorded from a spherical region of interest placed over known tumors with reference to $\mathrm{CT}$, $\left[{ }^{18} \mathrm{~F}\right]$ fluorodeoxyglucose-PET, and prior breast imaging studies, if applicable. The target lesion was the primary breast tumor. If already surgically removed $(n=1)$, the metastatic lesion with highest pretherapy $\mathrm{SUV}_{\max }$ was the target lesion for pre- and posttherapy measurements.

Statistics. No statistical analyses outside of calculation of average and median values were applied to the data set in this manuscript.

Study approval. Studies were approved by the University of Pennsylvania Institutional Review Board (numbers 826390 and 832165), and written informed consent was obtained from participants.

\section{Author contributions}

ESM was responsible for designing research studies, conducting experiments, acquiring data, analyzing data, and writing the manuscript; ARP was responsible for conducting experiments, acquiring data, analyzing data, and writing the manuscript; PDS was responsible for acquiring data and manuscript review; DAP was responsible for acquiring data and manuscript review; ASC was responsible for acquiring data and manuscript review; RKD was responsible for analyzing data and writing the manuscript; MDF was responsible for acquiring data and manuscript review; and SDC was responsible for designing research studies, conducting experiments, acquiring data, analyzing data, providing reagents, and writing the manuscript.

\section{Acknowledgments}

Support for this study was provided by Metavivor Translational Research Award (to EM, PDS, SDC), Susan G. Komen for the Cure CCR 16376362 (to EM), Department of Defense BC190315 (to EM), National Cancer Institute/NIH P30 CA016520 (to EM), and the Abramson Cancer Center Breakthrough Bike Challenge (to EM, ASC). We wish to thank the patients in this study for their participation and Meryl Weinreb, a Komen Patient Advocate in Science, for helpful discussions and study review. We thank Robert Mach, Britton Chance Professor of Radiology, University of Pennsylvania, for serving as a scientific advisor for this study. We are grateful to the Penn Medicine Cyclotron Facility and Hsiaoju Lee for radiotracer production and Kuiying $\mathrm{Xu}$ for chemical synthesis of the tracer precursor. We would like to thank colleagues at the University of Pennsylvania, Erin K. Schubert, Anthony Young, and Theresa E. Berger, for help with data collection. Mary Leonard and Katie Fine assisted with graphic design.

Address correspondence to: Elizabeth McDonald, Hospital of the University of Pennsylvania, 3400 Spruce Street/1 Silverstein, Philadelphia, Pennsylvania 19104-4283, USA. Email: elizabeth.mcdonald@ pennmedicine.upenn.edu.

1. Robson M, et al. Olaparib for metastatic breast cancer in patients with a germline BRCA mutation. NEngl J Med. 2017;377(6):523-533.

2. McCabe N, et al. Deficiency in the repair of DNA damage by homologous recombination and sensitivity to poly(ADP-ribose) polymerase inhibition. Cancer Res. 2006;66(16):8109-8115.

3. Rugo HS, et al. Adaptive randomization of veliparib-carboplatin treatment in breast cancer. N Engl J Med. 2016;375(1):23-34.

4. Zhou D, et al. Synthesis, [(1)(8)F] radiolabeling, and evaluation of poly (ADP-ribose) polymerase-1 (PARP-1) inhibitors for in vivo imaging of PARP-1 using positron emission tomography. Bioorg Med Chem. 2014;22(5):1700-1707.

5. Michel LS, et al. PET of poly (ADP-ribose) polymerase activity in cancer: preclinical assessment and first in-human studies. Radiology. 2017;282(2):453-463.

6. Makvandi M, et al. A radiotracer strategy to quantify PARP-1 expression in vivo provides a biomarker that can enable patient selection for PARP inhibitor therapy. Cancer Res. 2016;76(15):4516-4524.

7. Makvandi M, et al. A PET imaging agent for evaluating PARP-1 expression in ovarian cancer. J Clin Invest. 2018;128(5):2116-2126.

8. Pettitt SJ, et al. A genetic screen using the PiggyBac transposon in haploid cells identifies Parp1 as a mediator of olaparib toxicity. PLoS One. 2013;8(4):e61520.

9. Hopkins TA, et al. PARP1 trapping by PARP inhibitors drives cytotoxicity in both cancer cells and healthy bone marrow. Mol Cancer Res. 2019;17(2):409-419.

10. McDonald ES, et al. Positron emission tomography imaging of poly-(adenosine diphosphate-ribose) polymerase 1 expression in breast cancer: a nonrandomized clinical trial. JAMA Oncol. 2020;6(6):921-923. 